\title{
Time-dependent density functional theory study of squaraine dye-sensitized solar cells
}

\author{
Dario Rocca ${ }^{\mathrm{a}, \mathrm{b}, 1}$, Ralph Gebauer ${ }^{\mathrm{b}, \mathrm{c}}$, Filippo De Angelis ${ }^{\mathrm{d}, *}$, Mohammad K. Nazeeruddin ${ }^{\mathrm{e}}$, Stefano Baroni ${ }^{\mathrm{a}, \mathrm{b}}$ \\ a SISSA - Scuola Internazionale Superiore di Studi Avanzati, Via Beirut 2-4, I-34014 Trieste, Italy \\ ${ }^{\mathrm{b}}$ CNR-INFM DEMOCRITOS National Simulation Center, Trieste, Italy \\ ${ }^{c}$ ICTP - The Abdus Salam International Centre for Theoretical Physics, Strada Costiera 11, I-34014 Trieste, Italy \\ ${ }^{\mathrm{d}}$ Istituto CNR di Scienze e Tecnologie Molecolari (ISTM-CNR) and UdR INSTM Perugia, c/o Dipartimento di Chimica, Università di Perugia, I-06123 Perugia, Italy \\ ${ }^{\mathrm{e}}$ Laboratory for Photonics and Interfaces, Institute of Chemical Sciences and Engineering, School of Basic Sciences, Swiss Federal Institute of Technology, \\ CH-1015 Lausanne, Switzerland
}

\section{A R T I C L E I N F O}

\section{Article history:}

Received 18 February 2009

In final form 8 May 2009

Available online 13 May 2009

\begin{abstract}
A B S T R A C T
We employ time-dependent density functional theory to study the optical properties of a squaraine sensitized $\mathrm{TiO}_{2}$ system, as a model for the corresponding dye-sensitized solar cell. The all-organic squaraine dye is particularly promising for light absorption in the red spectral region. We compute the photoabsorption spectrum of a periodic $\mathrm{TiO}_{2}$ slab exposing anatase (101) surfaces, both for the clean slab and for the surface covered by the squaraine dye. Those spectra are compared to recent experimental data. The main absorption peaks are analyzed, concluding that both direct and indirect charge injection mechanisms are active in this system.
\end{abstract}

(c) 2009 Elsevier B.V. All rights reserved.

\section{Introduction}

In recent years Dye Sensitized Solar Cells (DSSCs) are attracting considerable interest. On the one hand, application oriented research is focused on the prospect of converting sunlight into electricity both with high efficiency and low costs [1]. On the other hand, fundamental theoretical research is unraveling the fast charge transfer mechanisms which underlie the functioning of such devices [2]. DSSCs consist of an overlayer of dye molecules adsorbed on the surface of a large gap semiconductor, typically $\mathrm{TiO}_{2}$ in the form of nanoparticles or thin films. The key process that allows for the harvesting of energy from the sun is the injection of light-excited electrons from the dye into the conduction band of the semiconductor substrate. The efficiency of this process depends on the structure of the dye/semiconductor interface, as well as on the precise nature of the electronic excited states involved in it. In general, the HOMO (highest occupied molecular orbital) of dye-sensitized $\mathrm{TiO}_{2}$ is localized on the dye, whereas the LUMO (lowest unoccupied molecular orbital) is localized on the substrate; the net effect is basically a lowering of the energy gap of the bare semiconductor substrate. Given this alignment of the energy levels, the injection of a photo-excited electron into the semiconductor can be due to different underlying mechanisms. Indeed,

\footnotetext{
* Corresponding author.

E-mail address: filippo@thch.unipg.it (F. De Angelis).

1 Present address: Department of Chemistry, University of California, Davis, Davis, California 95616, USA.
}

a direct transition can occur from the occupied levels of the dye to the conduction band of the $\mathrm{TiO}_{2}$ substrate (direct mechanism). In a second possible scenario an electron is firstly photo-excited to a dye localized orbital and then transferred to the semiconductor conduction band. This indirect mechanism is in general more efficient since it can involve a larger number of final states in the $\mathrm{TiO}_{2}$ conduction band. In this case, in order to achieve high electrontransfer quantum yields, light must produce long-lived excited states in the dye that are strongly coupled with a large number of states of same energy in the $\mathrm{TiO}_{2}$ conduction band. After the injection process, the photo-excited electron diffuses away into the substrate, thus determining a potential drop that produces electric power upon closure of the circuit through an electrolyte and a counter-cathode (the anode being the $\mathrm{TiO}_{2}$ substrate itself, in contact with a conducting collecting plate), while the charge neutrality and chemical stability of the dye is assured by the donation of an electron from the electrolyte.

A breakthrough of the DSSC technology has been made possible by the extraordinary performance of ruthenium complexes sensitizer attached to nanocrystalline $\mathrm{TiO}_{2}$ films $[1,3,4]$. Such transition metal complexes are still the most widespread dyes in this kind of application, but research to find alternative optimal dyes is very active. Ruthenium complexes absorb visible light mainly in the green region, around $2.3 \mathrm{eV}$. For this reason, in order to further improve the performance of DSSCs, it is necessary to better fit the absorption band of the dye with the solar spectrum, which extends into the red and infra-red region. The introduction of dyes that enhance absorption in the red/near infrared region is, therefore, of 
fundamental importance. From this point of view squaraines are good candidates because of their intense absorption in that energy range. Several pilot applications already exist [5-10]. In Ref. [10] a new asymmetrical squaraine has been introduced, with a carboxylic acid group to anchor the molecule to a $4 \mu \mathrm{m} \mathrm{TiO}{ }_{2}$ film. The DSSC built with this molecule reached an Incident Photon to Current Efficiency (IPCE) of $80 \%$ at about $1.9 \mathrm{eV}$, up to now one of the best performances for these devices in the far-red/infrared region.

In spite of the large amount of experimental work on DSSC's, a theoretical understanding of such devices is still limited by practical and conceptual difficulties. The main theoretical challenge concerning DSSCs is to understand the mechanisms ruling their functioning at the atomic scale, with the final purpose of engineering new dyes with custom-tailored optimal properties. To that purpose an accurate description of the excited state properties of the combined dye/semiconductor system is necessary. TDDFT is a suitable approach to address this issue and some applications of this method to DSSCs already exist [11-14]. Indeed, TDDFT allows for an approximate account of correlation effects on the spectra, at a substantially lower computational price than approaches based on many-body perturbation theory. In spite of this, the simulation of extended portions of the spectrum (as opposed to the calculation of a few individual lines) of fairly large and complex systems, such as those needed to model a DSSC, still remains a major computational challenge.

\section{Computational method and discussion of the results}

A particularly efficient implementation to solve TDDFT equations for large systems has been recently introduced in Refs. [2022 ]. Within this approach the dynamical polarizability of an interacting electron system is expressed as an appropriate off-diagonal matrix element of the resolvent of a Liouvillian superoperator. By using a representation for operators and density matrices borrowed from density functional perturbation theory $[24,25,23]$ we can avoid the explicit calculation of the unoccupied states of the ground-state Hamiltonian. The problem is then solved through a method based on the non-symmetric Lanczos algorithm; furthermore an inexpensive extrapolation of the Lanczos coefficients can be used to enhance even more the convergence properties of this iterative chain [22]. The overall computational cost of this procedure can be compared to that of ground state calculations. This new technique has been implemented in a plane wave (PW) basis set within the QUANTUM ESPRESSO distribution of computer codes (http://www.quantum-espresso.org).

We applied this new approach to investigate the fundamental processes of dye-sensitized solar cells working in the red/near infrared region. We consider the specific case of the squaraine synthesized in Ref. [10], since it has been shown to be the more efficient in this kind of applications. Our model consists of the molecule adsorbed on a two layers periodically repeated $\mathrm{TiO}_{2}$ slab; the supercell used for our simulations is shown in Fig. 1. The structure of the squaraine was simplified by replacing the octyl substituents with methyls; this simplification does not lead to significant changes in the dye electronic structure [10]. The exposed surface of the slab is the $(101)$ of anatase structure, using a $(1 \times 4)$ primitive cell. The dimension of the cell containing the system is $19.35 \times 28.61 \times 54.28 \mathrm{a}_{0}^{3}$. We also performed the simulation of the isolated slab and of the isolated molecule in cells of dimension $19.35 \times 28.61 \times 45.66 \mathrm{a}_{0}^{3}$ and $52.91 \times 26.46 \times 23.62 \mathrm{a}_{0}^{3}$, respectively. This model system is chosen as a compromise in between the computational cost and the purpose of minimizing the spurious interactions between repeated dye images along the directions parallel to the surface, i.e. $x$ and $y$. In these directions, the distance

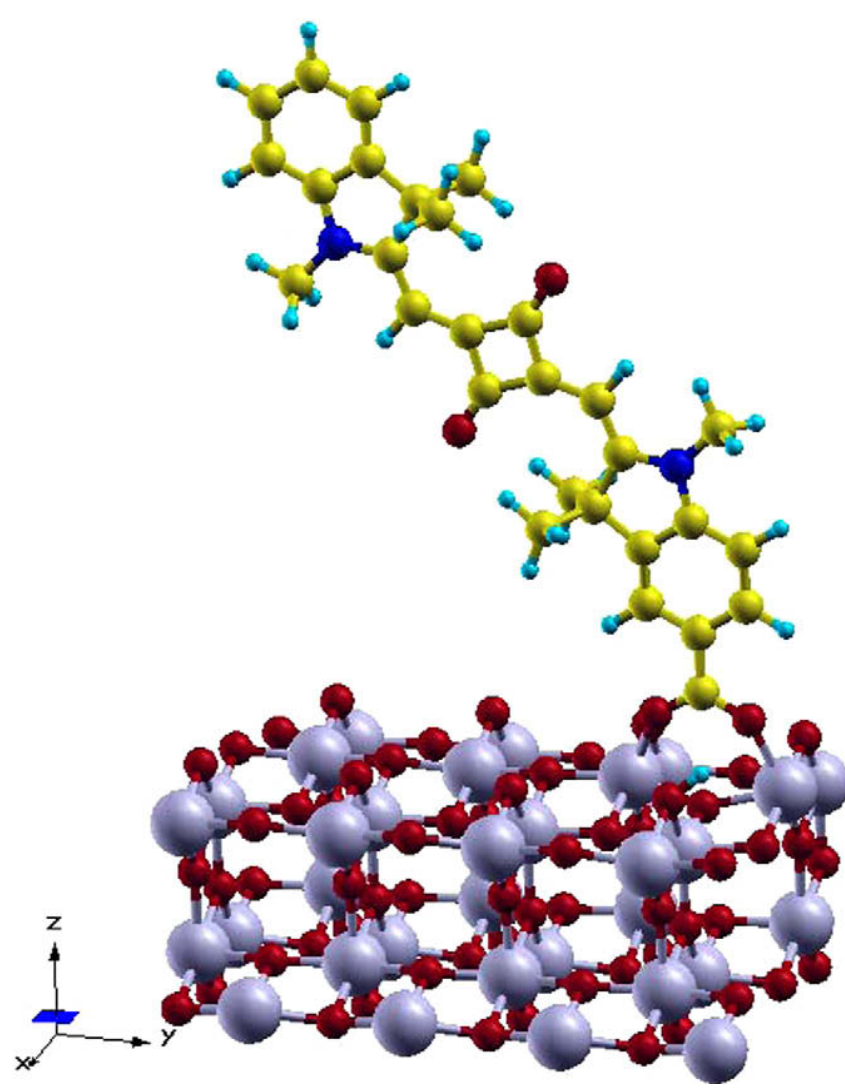

Fig. 1. Translational unit of the squaraine DSSC model, consisting of the molecule adsorbed on a two-layer slab. The system exposes the (101) surface and is oriented with the $z$ axis perpendicular to such surface plane. A proton, initially residing on the dye, is transferred to the surface upon geometry optimization.

between repeated images of the dye molecules is about $5 \AA$, which we have checked to be sufficient to decouple the interaction among periodic images. Also in the direction perpendicular to the slab, i. e. $z$, we have checked that the vacuum (ca. 6/AA) in between the lowermost $\mathrm{TiO}_{2}$ layer and the repeated dye image is sufficient to minimize spurious dipole-dipole interactions among repeated images.

The calculations were performed sampling the $\Gamma$ point only in the first Brillouin zone. We used the Perdew-Burke-Ernzerhof (PBE) [15] XC-GGA functional, ultra-soft pseudopotentials [26] and a PW's basis set, up to a kinetic energy cut-off of $25 \mathrm{Ry}$ and $200 \mathrm{Ry}$ for the charge density. The absorption spectra are calculated as $I(\omega) \propto \omega \operatorname{Im}(\bar{\alpha}(\omega))$, where $\bar{\alpha}$ is the spherical average (average of the diagonal elements) of the dipole polarizability; an imaginary part of 0.002 Ry has been added to the frequency, in order to smooth the divergences of the polarizability.

To check the adequacy of the GGA functional to reproduce the electronic properties of the dye, we performed additional TDDFT calculations on the squaraine system by the GaUSSIAN 03 program [27] using the same PBE functional used in the reported results and the B3LYP [28] functional. In doing so we selected the rather large $6-311 \mathrm{G}^{*}$ basis set. Compared to the experimental value of $1.94, \mathrm{PBE} / 6-311 \mathrm{G}^{*}$ results provide a $2.07 \mathrm{eV}$ transition, in excellent agreement with the experiment and with periodic results reported here, while B3LYP provides a main transition at $2.27 \mathrm{eV}$, blueshifted compared to the experimental value by as much as $0.31 \mathrm{eV}$.

As a preliminary study we ascertained the proper alignment of the molecular electronic states with those of the substrate and calculated the projected density of Kohn-Sham states (PDOS) for the full DSSC system. The results showing the PDOS are reported in 


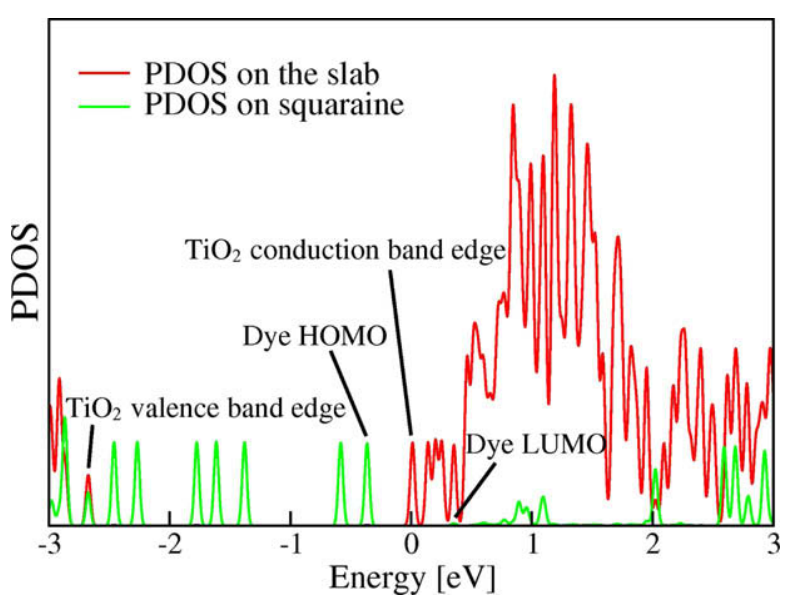

Fig. 2. Projected density of states (PDOS) for the combined dye-semiconductor system. $\mathrm{TiO}_{2}$ (dye) states are in red (green). (For interpretation of the references to colour in this figure legend, the reader is referred to the web version of this article.) a

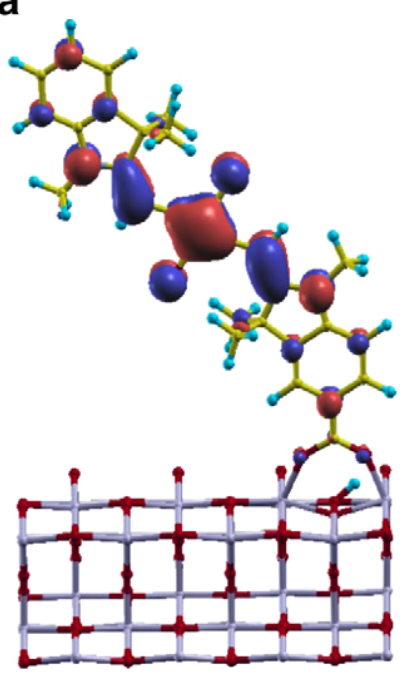

b

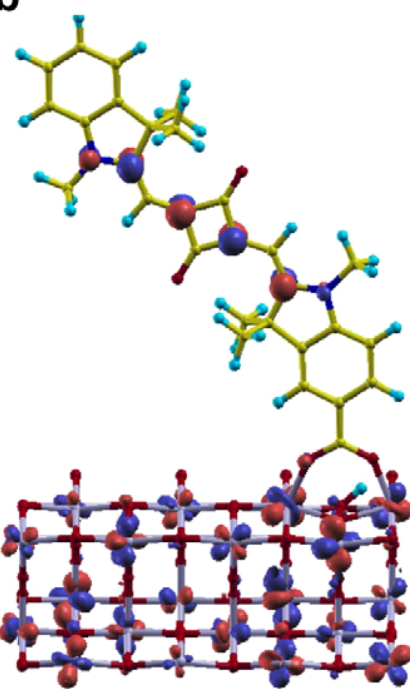

Fig. 3. Isosurfaces of the HOMO (a) and LUMO+34 (b) states.

Fig. 2, where we also indicated the positions of the $\mathrm{TiO}_{2}$ valence and conduction band edges along with the energies of the dye HOMO and LUMO. We obtained the expected behavior for dye-sensitized $\mathrm{TiO}_{2}$ : the HOMO corresponds to a purely dye state (Fig. 3a), well separated from $\mathrm{TiO}_{2}$ states, while the LUMO corresponds to a state of the slab (not shown). We also notice that the dye LUMOs are energetically located in a region of high density of $\mathrm{TiO}_{2}$ conduction band states, a fact that should lead to a strong coupling between the dye and the semiconductor states, thus facilitating electron injection.

For this reason molecular states tend to hybridize with the states of the slab. Mixing is also increased by proton transfer from the protonated carboxylic anchoring group of the squaraine dye to $\mathrm{TiO}_{2}$, which takes place in our system upon geometry optimization [14]. Indeed, the analysis of the isodensities of the KS orbitals in conduction band has revealed sizable mixing of dye and $\mathrm{TiO}_{2}$ states (the state LUMO+34 in Fig. 3b is an example of such behaviour).

We move now to the optical properties of our dye-sensitized $\mathrm{TiO}_{2}$ system. A proper description of the optical threshold of the $\mathrm{TiO}_{2}$ substrate is crucial in order for our model to mimic the optical properties of the real system. Both the KS HOMO-LUMO gap and

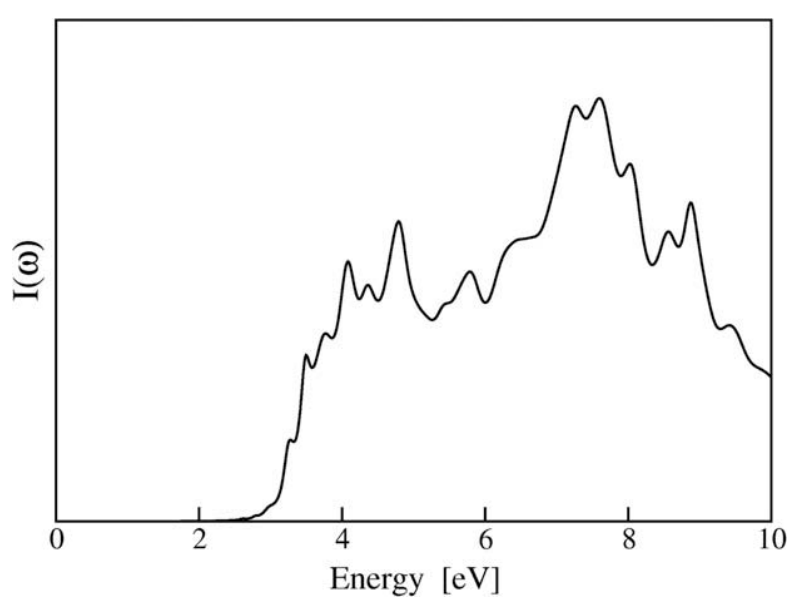

Fig. 4. Absorption spectrum of the $\mathrm{TiO}_{2}$ slab, as calculated from TDDFT between 0 and $10 \mathrm{eV}$.

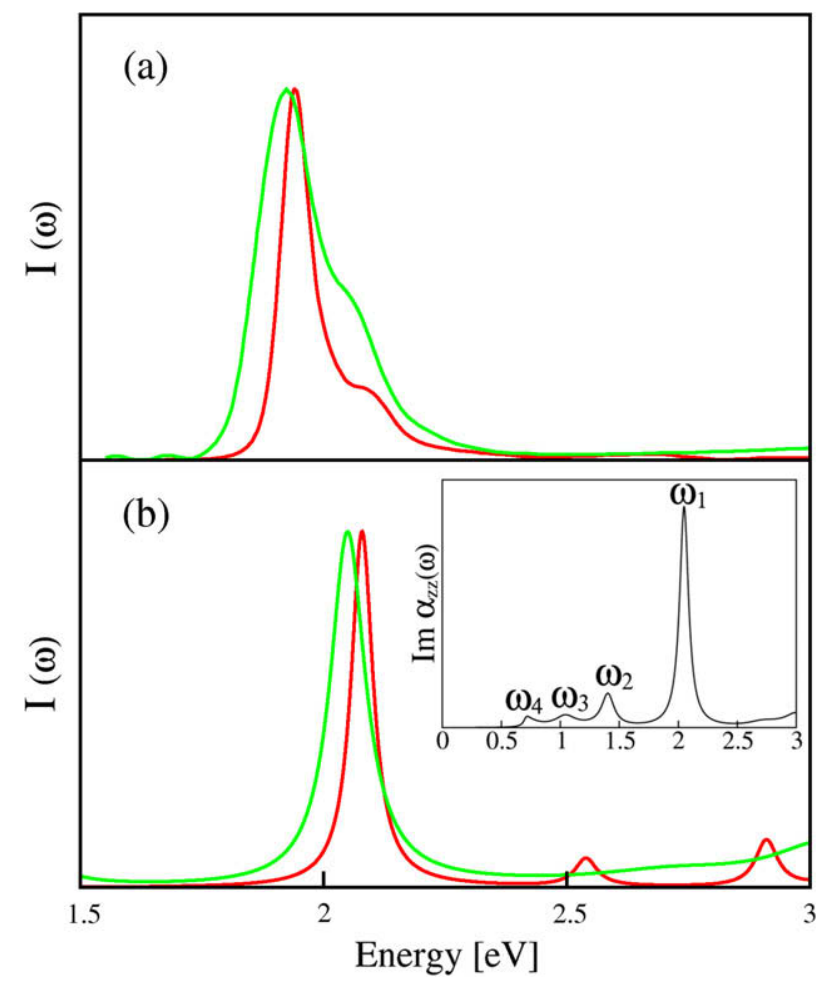

Fig. 5. (a) Experimental absorption spectrum of squaraine in ethanol (red) and adsorbed on a $4 \mu \mathrm{mTiO}_{2}$ film (green). (b) TDDFT results for the absorption spectrum of squaraine in gas-phase (red) and adsorbed on a $\mathrm{TiO}_{2}$ slab (green). For comparative purposes the green curve has been multiplied by a factor 1.2. In the inset the imaginary part of the $z z$ component of the polarizability is shown in the energy range between 0 and $3 \mathrm{eV}$. (For interpretation of the references to colour in this figure legend, the reader is referred to the web version of this article.)

the TDDFT optical gap are underestimated to the value of $2.5 \mathrm{eV}$, as a consequence of the well known DFT-GGA gap problem [16]. Nevertheless the overall shape of the TDDFT absorption spectrum in Fig. 4 shows a very low intensity in the visible region and makes this model substantially reliable in the description of a realistic $\mathrm{TiO}_{2}$ substrate. We notice that this problem is ameliorated by hybrid functionals, which usually deliver improved band gaps compared to GGA functionals $[11,17,18]$.

As shown in Fig. 5, experimentally the squaraine dye presents a strong absorption peak around $1.94 \mathrm{eV}$ in the visible region [10], 
which corresponds to a $\pi \rightarrow \pi^{*}$ transition involving substantial charge-transfer from the squaraine core to the molecular region where the carboxylic groups are located. This excited state is potentially strongly coupled to the semiconductor conduction band, due to charge delocalization involving the anchoring carboxylic group. When the molecule is adsorbed on a $4 \mu \mathrm{m} \mathrm{TiO}{ }_{2}$ film the spectral features do not significantly change, but the main peak is broadened and the maximum in the absorption is slightly redshifted by about $0.02 \mathrm{eV}$, due to the interaction of the anchoring group with the surface. A shoulder of the main transition is present, probably due to vibronic transitions [9]. In Fig. 5, the TDDFT spectra are shown for a molecule in the gas phase and for our dye-on-a-slab model. The main transition of squaraine has been found at $2.08 \mathrm{eV}$, shifted only by $0.14 \mathrm{eV}$ compared to experimental results in Fig. 5. The calculated value is essentially the same as previously found in a GGA-TDDFT calculation employing a localized basis set [10]. This shift is possibly related to solvent effects, but the magnitude of the discrepancy is beyond the expected accuracy of this kind of TDDFT calculations. When the molecule is adsorbed on the surface, a small red-shift of about $0.03 \mathrm{eV}$ occurs, in close agreement with experimental results. The shoulders observed in the experimental spectra are not present in our results, possibly due to our neglect of vibronic effects. As in the experimental data, the calculated spectrum of the dye + slab system shows only a weak absorption intensity for energies beyond the first strong transition. This result confirms once more that the oscillator strength of the slab spectrum can be neglected for energies below $3 \mathrm{eV}$ (Fig. 4) and that the substrate model gives an accurate description of the real system.

We have shown that theoretical and experimental results in the visible range are in good agreement. Both seem to suggest an indirect transition mechanism in the solar cell, since there is no significant change between the spectrum of free-standing and adsorbed molecules. Nevertheless in the TDDFT results some weak transition appeared in the infrared region, namely for energies lower than the main transition peak. In this energy region no experimental data are available for comparison. These transitions are particularly evident in the $z z$ component of the absorption (see inset of Fig. 5), but with smaller intensity in the $y y$ component as well. Despite their weakness, these transitions can bring a contribution to the solarto-electric power conversion mechanism.

To investigate in detail the origin of these transitions, we calculated the charge linear response of the system to the application of an external electric field $E_{z}$ along the $z$ direction (since we are interested in the $z z$ component of the polarizability):

$n_{E_{z}}^{\prime}(\mathbf{r}, \omega)=\int \chi\left(\mathbf{r}, \mathbf{r}^{\prime}, \omega\right) z^{\prime} d \mathbf{r}^{\prime}$

where $\chi\left(\mathbf{r}, \mathbf{r}^{\prime}, \omega\right)$ is the generalized susceptibility; $n_{E_{z}}^{\prime}(\mathbf{r}, \omega)$ is useful to show the spatial localization of the dipoles induced by the field $E_{z}$ at a given frequency $\omega$. We computed explicitly the integral of this function in the planes orthogonal to the $z$ direction

$\bar{n}_{E_{z}}^{\prime}(z, \omega)=\frac{1}{\sum_{x y}} \int n_{E_{z}}^{\prime}(\mathbf{r}, \omega) d x d y$,

which gives information on the dipoles along $z$. Indeed Eq. (2) is related to the element $\alpha_{z z}$ of the polarizability tensor through

$\alpha_{z z}(\omega)=\int \bar{n}_{E_{z}}^{\prime}(z, \omega) z d z$.

In principle this relation is ill-defined in periodic boundary conditions, since it depends on the choice of the simulation cell [19]. Nevertheless, at least in the energy range we are considering, the orbitals involved are far from the boundaries of the supercell along $\mathrm{z}$ and for practical purposes there is no substantial difference with

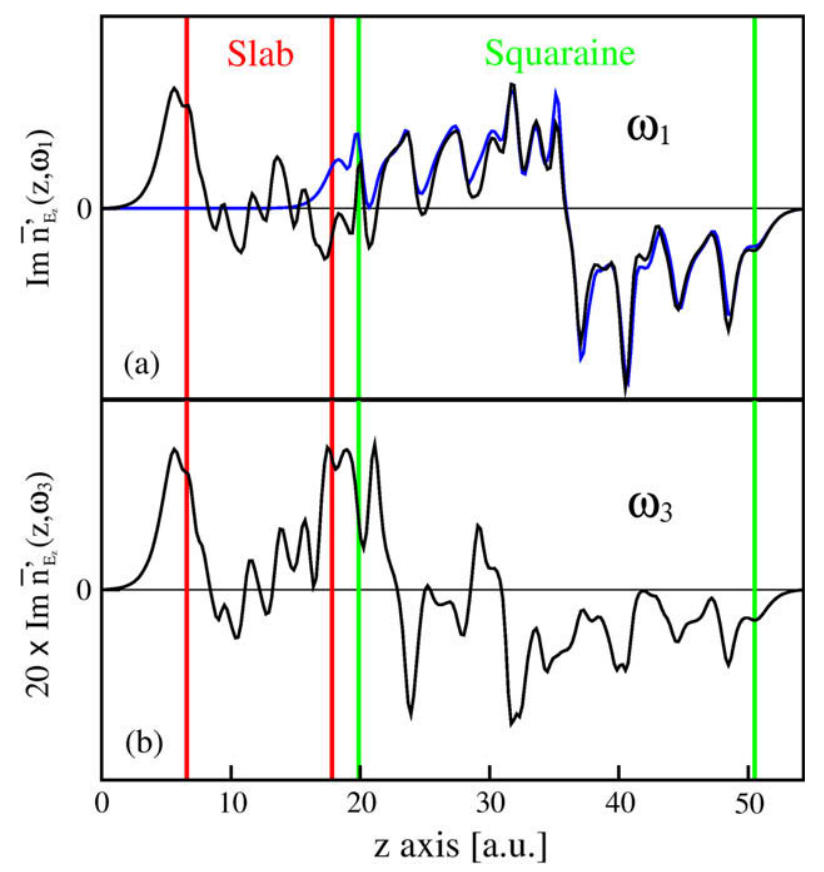

Fig. 6. Imaginary part of the charge response (2) for the DSSC system as a function of the $z$ coordinate in correspondence to the energy $\omega_{1}$. In blue the same quantity is shown for the molecule standing alone; for comparative purposes this curve has been divided by 1.2. The charge response for the transition $\omega_{3}$ is shown in the frame (b). (For interpretation of the references to colour in this figure legend, the reader is referred to the web version of this article.)

respect to an open boundary conditions approach. Eq. (3) has been verified to hold $a$ posteriori (namely by computing the absorption spectrum starting from the charge density response function).

In Fig. 6 we show the values of $\operatorname{Im} \bar{n}_{E_{z}}^{\prime}(z, \omega)$ for two of the transitions displayed in Fig. 6 as a function of the $z$ coordinate; the region of space occupied by the molecule and by the slab are delimited by green and red lines respectively. In the frame (a) we see a strong dipole in the dye region at energy $\omega_{1}$, which is very similar to what we have found for the free-molecule (blue line); almost no charge response is present in the region of the slab, but for a charge accumulation opposite to the one where the dye is anchored. In the other frames of Fig. 6 the charge response function is shown for $\omega_{3}$; in this case it is evident that this low energy peak is mainly associated to a molecule-slab transition. Indeed the density response is almost exclusively negative in the squaraine region and almost exclusively positive in the slab and the slab-dye interface region, thus forming a net dipole between the molecule and the slab. This transition corresponds to a direct injection mechanism.

The TDDFT analysis of the optical spectra and of the charge response functions indicates that the functioning of the squaraine solar cell is dominated by an indirect injection mechanism, even though a weak contribution arising from a direct dye to semiconductor transition cannot be excluded. Further work is necessary to improve the reliability of TDDFT calculations on DSSC systems, especially concerning the description of the semiconductor. A more realistic model with many layers would lead to a more appropriate description of such system; but the most important problem concerns the correction of the gap of $\mathrm{TiO}_{2}$, that we have underestimated in our calculations. To this purpose, hybrid functionals, despite a large computational cost, are a suitable method to correct both the KS gap and the TDDFT optical gap of the substrate [11]. Nevertheless, our computational approach is able to provide considerable insight into the electronic structure and the nature of the excited states of this important class of systems, bridging 
chemistry and solid state physics, thus aiding the design of new and more efficient dyes and DSSC devices.

\section{Acknowledgements}

We thank the DEISA Consortium (www.deisa.eu), co-funded through the EU FP6 project RI-031513 and the FP7 project RI222919, for support within the DEISA Extreme Computing Initiative. F.D.A. thanks MIUR (FIRB 2003: Molecular compounds and hybrid nanostructured materials with resonant and non resonant optical properties for photonic devices) and INSTM (PRISMA 2007) for financial support.

\section{References}

[1] B. O'Regan, M. Grätzel, Nature 53 (1991) 737.

[2] W. Duncan, O. Prezhdo, Ann. Rev. Phys. Chem. 58 (2007) 143.

[3] M.K. Nazeeruddin et al., J. Am. Chem. Soc. 115 (1993) 6382.

[4] M. Grätzel, Nature 414 (2001) 338.

[5] C. Li, W. Wang, X. Wang, B. Zhang, Y. Cao, Chem. Lett. 24 (2005) 554.

[6] S. Alex, U. Santhosh, S.J. Das, J. Photochem. Photobiol. A 172 (2005) 65

[7] Y. Chen, Z. Zeng, C. Li, W. Wang, X. Wang, B. Zhang, New J. Chem. 29 (2005) 773.
[8] A. Otsuka, K. Funabiki, N. Sugiyama, T. Yoshida, M. Minoura, M. Matsui, Chem. Lett. 35 (2006) 666

[9] A. Burke, L. Schmidt-Mende, S. Ito, M. Grätzel, Chem. Commun. (2007) 234.

[10] J.-H. Yum et al., J. Am. Chem. Soc. 129 (2007) 10320.

[11] F. De Angelis, A. Tilocca, A. Selloni, J. Am. Chem. Soc. 126 (2004) 15024.

[12] P. Persson, M.J. Lundqvist, J. Phys. Chem. B 109 (2005) 11918.

[13] K.J. Lundqvist, M. Nilsing, S. Lunell, B. Kemark, P. Persson, J. Phys. Chem. B 110 (2006) 20513.

[14] F. De Angelis, S. Fantacci, A. Selloni, M.K. Nazeeruddin, M. Grätzel, J. Am. Chem. Soc. 129 (2007) 14156

[15] J.P. Perdew, K. Burke, M. Ernzerhof, Phys. Rev. Lett. 77 (1996) 3865.

[16] C. Stampfl, C.G. Van de Walle, Phys. Rev. B 59 (1999) 5521.

[17] J. Muscat, A. Wander, N.M. Harrison, Chem. Phys. Lett. (2001). 324, 397

[18] C. Di Valentin, G. Pacchioni, A. Selloni, Phys. Rev. Lett. (2006) 166803.

[19] R. Resta, Rev. Mod. Phys. 66 (1994) 899.

[20] B. Walker, A.M. Saitta, R. Gebauer, S. Baroni, Phys. Rev. Lett. 96 (2006) 113001.

[21] B. Walker, R. Gebauer, J. Chem. Phys. 127 (2007) 164106.

[22] D. Rocca, R. Gebauer, Y. Saad, S. Baroni, J. Chem. Phys. 128 (2008) 154105.

[23] S. Baroni, S. de Gironcoli, A. Dal Corso, P. Giannozzi, Rev. Mod. Phys. 73 (2001) 515.

[24] S. Baroni, P. Giannozzi, A. Testa, Phys. Rev. Lett. 58 (1987) 1861

[25] X. Gonze, Phys. Rev. A 52 (1995) 1096.

[26] D. Vanderbilt, Phys. Rev. B 41 (1990) 7892.

[27] M.J. Frisch, et al., Gaussian 03, Revision D.1, Gaussian, Inc., Pittsburgh PA, 2003.

[28] A.D. Becke, J. Chem. Phys. 98 (1993) 5648. 